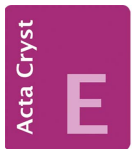

CRYSTALLOGRAPHIC COMMUNICATIONS

ISSN 2056-9890
Received 4 June 2018

Accepted 25 June 2018

Edited by C. Rizzoli, Universita degli Studi di Parma, Italy

Keywords: crystal structure; pyridiniminium salt; hydrogen bonding; Hirshfeld surface analysis.

CCDC reference: 1851334

Supporting information: this article has supporting information at journals.iucr.org/e
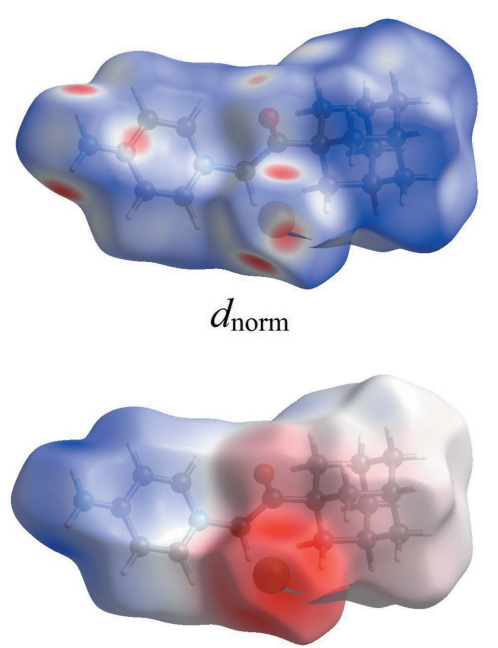

Electrostatic Potential

\section{Crystal structure and Hirshfeld surface analysis of a pyridiniminium bromide salt: 1-[2-(adamantan-1- yl)-2-oxoethyl]pyridin-4-iminium bromide}

\author{
Huey Chong Kwong, ${ }^{a}$ Imdad Mahmud Pathi, ${ }^{\text {b }}$ C. S. Chidan Kumar, ${ }^{\text {c* }}$ Ching Kheng \\ Quah $^{\text {b }}$ and Md. Azharul Arafath ${ }^{\text {d* }}$
}

\author{
${ }^{a}$ School of Chemical Sciences, Universiti Sains Malaysia, 11800 USM, Penang, ${ }^{\mathbf{b}}$ X-ray Crystallography Unit, School of \\ Physics, Universiti Sains Malaysia, 11800 USM, Penang, Malaysia, '⿳Department of Engineering Chemistry, Vidya Vikas \\ Institute of Engineering \& Technology, Visvesvaraya Technological University, Alanahalli, Mysuru 570028, Karnataka, \\ India, and ${ }^{\mathbf{d}}$ Department of Chemistry, Shahjalal University of Science and Technology, Sylhet 3114, Bangladesh. \\ *Correspondence e-mail: chidankumar@gmail.com, arafath_sustche90@yahoo.com
}

In the cation of the title salt, $\mathrm{C}_{17} \mathrm{H}_{23} \mathrm{~N}_{2} \mathrm{O}^{+} \cdot \mathrm{Br}^{-}$, the adamantyl moiety and the pyridiniminium ring are inclined to the ketone bridge by torsion angles of $-78.1(2)(\mathrm{C}-\mathrm{C}-\mathrm{C}=\mathrm{O})$ and $58.3(2)^{\circ}(\mathrm{C}-\mathrm{C}-\mathrm{N}-\mathrm{C})$, respectively, and the ketone bridge has a $\mathrm{C}-\mathrm{C}-\mathrm{C}-\mathrm{N}$ torsion angle of $174.80(15)^{\circ}$. In the crystal, the cations are connected into chains parallel to the $c$ axis by $\mathrm{C}-\mathrm{H} \cdots \mathrm{O}$ hydrogen bonds. The chains are further linked into layers parallel to the $b c$ plane by $\mathrm{N}-\mathrm{H} \cdots \mathrm{Br}$ and $\mathrm{C}-\mathrm{H} \cdots \mathrm{Br}$ hydrogen bonds, $\mathrm{C}-\mathrm{H} \cdots \pi$ interactions and $\pi-\pi$ stacking interactions [centroid-to-centroid distance $=3.5657$ (11) $\AA$ ]. A Hirshfeld surface analysis, which comprises the $d_{\text {norm }}$ surface, electrostatic potential map and two-dimensional fingerprint plots, was carried out to verify the contribution of the various intermolecular interactions.

\section{Chemical context}

Adamantane derivatives have been shown to exhibit various biological activities such as antiviral (Zoidis et al., 2010), antidiabetic (Zettl et al., 2010), antimicrobial (Piérard et al., 2009), anti-inflammatory (Lamanna et al., 2012), antioxidant (Priyanka et al., 2013) and central nervous system activities (Reisberg et al., 2003). Besides, adamantane-based chemotherapeutics have been developed for treating viral infections, for example influenza A, herpes simplex and HIV (Liu et al., 2011). There are a number of negatively charged enzymes and cofactors and many diseases, including cystic fibrosis, have been found to result from defects in the ion channel function (Ashcroft, 1999). The anion- $\pi$ non-covalent interaction has been explored both theoretically and experimentally and selective anion receptors and channels have been designed (Ballester, 2008; Schottel et al., 2008; Hay \& Bryantsev, 2008; Frontera et al., 2011).

Ionic liquids (ILs) have attracted a lot of interest over the past decade because of their unusual range of properties such as negligible vapour pressure, excellent thermal stability in a wide temperature range, no flammability and high ionic conductivity (Davis, 2004). ILs are excellent alternatives to volatile organic compounds (VOCs). An ionic liquid has a strong solvation ability and can dissolve polar and non-polar species with efficient selectivity, which can be modified by 
changing the anion (Blanchard et al., 2001). ILs have been used successfully as solvents in several reactions such as isomerization, dimerization, hydrogenation, and Heck and Suzuki coupling reactions (Chauvin \& Olivier-Bourbigou, 1995; Holbrey \& Seddon, 1999). They have also performed well as solvents in bio-catalysed and homogeneous catalytic reactions, and can be used as lubricants to wet the surface of metals, polymers and inorganic materials (Crosthwaite et al., 2004).

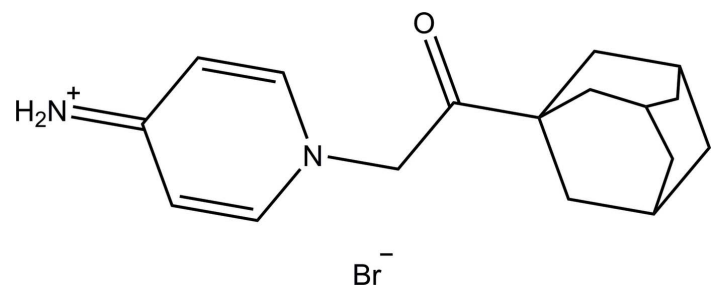

\section{Structural commentary}

Fig. 1 shows the asymmetric unt of the title salt, which consists of a 1-[2-(adamantan-1-yl)-2-oxoethyl]pyridin-4-iminium cation and a bromide anion. The cation is constructed from an adamantyl moiety $(\mathrm{C} 1-\mathrm{C} 10)$ and a pyridiniminium ring (N1/ C13-C17), which are connected by a ketone bridge $[(\mathrm{C} 11=\mathrm{O} 1)-\mathrm{C} 12]$. The bond angles formed by the quaternary carbon (C1) with the surrounding secondary carbons (C2, C6 and C7) are comparable with those reported for related structures which range from 107.40 (12) to $110.82(13)^{\circ}$ (Rouchal et al., 2011). Both the adamantyl and pyridiniminium rings are twisted away from the ketone bridge to reduce repulsion, as indicated by the torsion angles $\mathrm{C} 6-$ $\mathrm{C} 1-\mathrm{C} 11=\mathrm{O} 1 \quad\left[-78.1(2)^{\circ}\right]$ and $\mathrm{C} 11-\mathrm{C} 12-\mathrm{N} 1-\mathrm{C} 13$ $\left[58.3(2)^{\circ}\right]$. The ketone bridge is in an antiperiplanar conformation $\left[\mathrm{C} 1-\mathrm{C} 11-\mathrm{C} 12-\mathrm{N} 1=174.80(15)^{\circ}\right]$. The dihedral angle formed by the pyrimidinium ring with the ketone bridge is $59.77(14)^{\circ}$. Bond lengths and angles in the cation are within normal ranges (Allen, 2002). However, the $\mathrm{N} 2-\mathrm{C} 15$ bond length [1.325 (2) $\AA$ ] is shorter than expected for an $\mathrm{NH}_{2}-\mathrm{C}_{\mathrm{ar}}$ single bond [1.38 (3) §]], indicating partial double-bond character. Similar bond lengths are found in related compounds with an $\mathrm{N}^{+}=\mathrm{C}$ double bond (Chidan Kumar et al., 2017; Sharmila et al., 2014; Yue et al., 2013).

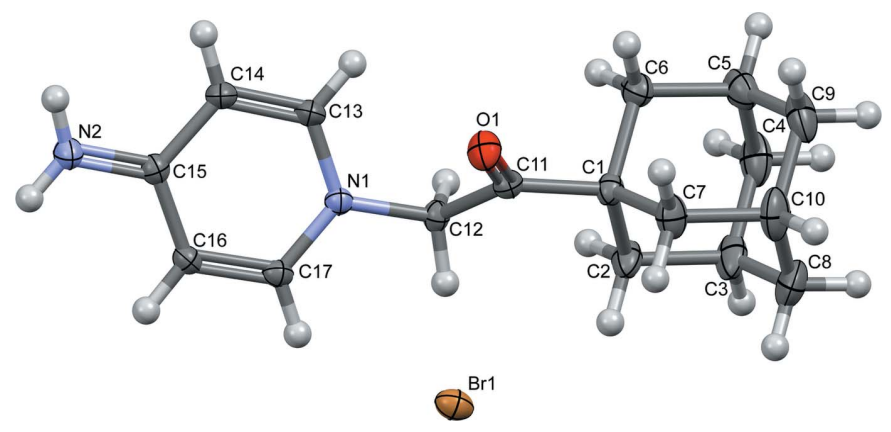

Figure 1

The molecular structure of the title salt with displacement ellipsoids drawn at the $50 \%$ probability level.
Table 1

Hydrogen-bond geometry $\left(\AA{ }^{\circ}\right)$.

$C g 1$ is the centroid of the $\mathrm{N} 1 / \mathrm{C} 13-\mathrm{C} 17$ ring.

\begin{tabular}{lllll}
\hline$D-\mathrm{H} \cdots A$ & $D-\mathrm{H}$ & $\mathrm{H} \cdots A$ & $D \cdots A$ & $D-\mathrm{H} \cdots A$ \\
\hline $\mathrm{N} 2-\mathrm{H} 1 N 2 \cdots \mathrm{Br} 1^{\mathrm{i}}$ & $0.84(2)$ & $2.73(2)$ & $3.499(2)$ & $153(2)$ \\
$\mathrm{N} 2-\mathrm{H} 2 N 2 \cdots \mathrm{Br} 1^{\text {ii }}$ & $0.85(2)$ & $2.56(2)$ & $3.393(2)$ & $169(2)$ \\
$\mathrm{C} 12-\mathrm{H} 12 A \cdots \mathrm{Br} 1^{\mathrm{iii}}$ & 0.97 & 2.72 & $3.664(2)$ & 166 \\
$\mathrm{C} 17-\mathrm{H} 17 A \cdots \mathrm{O} 1^{\text {iv }}$ & 0.93 & 2.59 & $3.434(2)$ & 150 \\
$\mathrm{C} 14-\mathrm{H} 14 A \cdots C g 1^{\mathrm{i}}$ & 0.93 & 2.94 & $3.608(2)$ & 130 \\
\hline
\end{tabular}

Symmetry codes: (i) $-x, y-\frac{1}{2},-z+\frac{1}{2}$; (ii) $-x,-y+1,-z+1$; (iii) $x, y-1, z$; (iv) $x,-y+\frac{1}{2}, z+\frac{1}{2}$.

\section{Supramolecular features}

In the crystal, the cations are linked into chains along the $c$ axis direction via $\mathrm{C} 17-\mathrm{H} 17 A \cdots \mathrm{O} 1$ hydrogen bonds (Table 1 , Fig. 2). The chains interact through $\mathrm{N}-\mathrm{H} \cdots \mathrm{Br}$ and $\mathrm{C}-$ $\mathrm{H} \cdots \mathrm{Br}$ hydrogen bonds to form layers parallel to the $b c$ plane, which are further enforced by $\mathrm{C}-\mathrm{H} \cdots \pi$ and $\pi-\pi$ interactions [centroid-to-centroid distance 3.5657 (11) $\mathrm{A}$ ].

\section{Hirshfeld Surface Analysis}

The Hirshfeld surface analysis (Spackman \& Jayatilaka, 2009) of the title salt was performed using CrystalExplorer3.1 (Wolff et al., 2012), and comprises $d_{\text {norm }}$ surface plots, electrostatic potentials and two-dimensional fingerprint plots (Spackman \& McKinnon, 2002). The ball-and-stick model, $d_{\text {norm }}$ surface and electrostatic potential plots of the title salt are shown in Fig. 3. Those plots were generated in order to quantify and give visual confirmation of the intermolecular interactions and to explain the observed crystal packing. The dark-red spots on the $d_{\text {norm }}$ surface arise because of short interatomic contacts, while the other weak intermolecular interactions appear as light-red spots. Furthermore, the negative electrostatic potential (red region) in the electrostatic potential map indicates hydrogen-acceptor potential, whereas the hydrogen donors are represented by positive electrostatic potential (blue region) (Spackman et al., 2008).

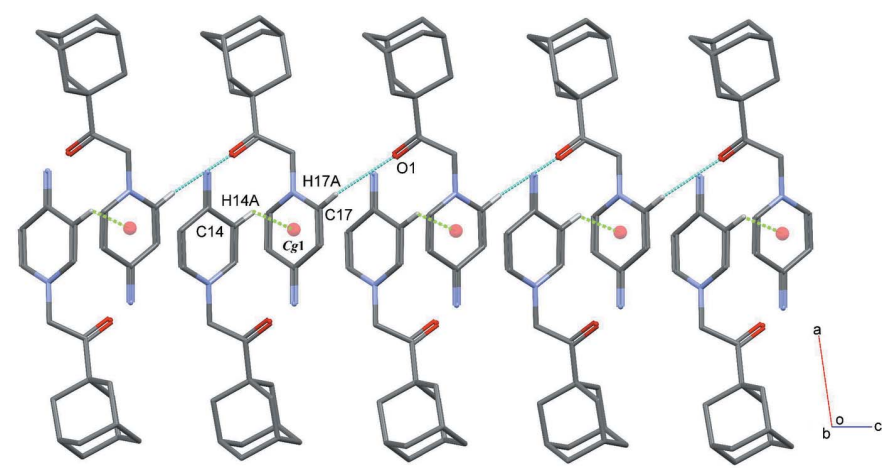

Figure 2

Partial packing diagram of the the cations showing the $\mathrm{C} 17-\mathrm{H} 17 A \cdots \mathrm{O} 1$ hydrogen bonds (blue dashed lines) and the $\mathrm{C} 14-\mathrm{H} 14 A \cdots \pi$ interactions (green dashed lines). 


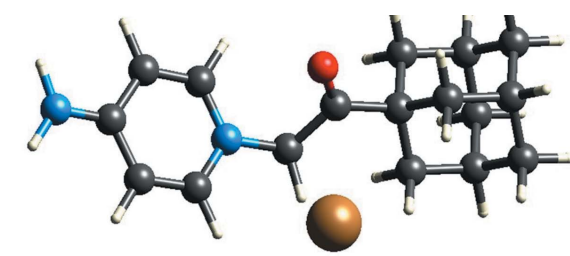

Ball and Stick Model
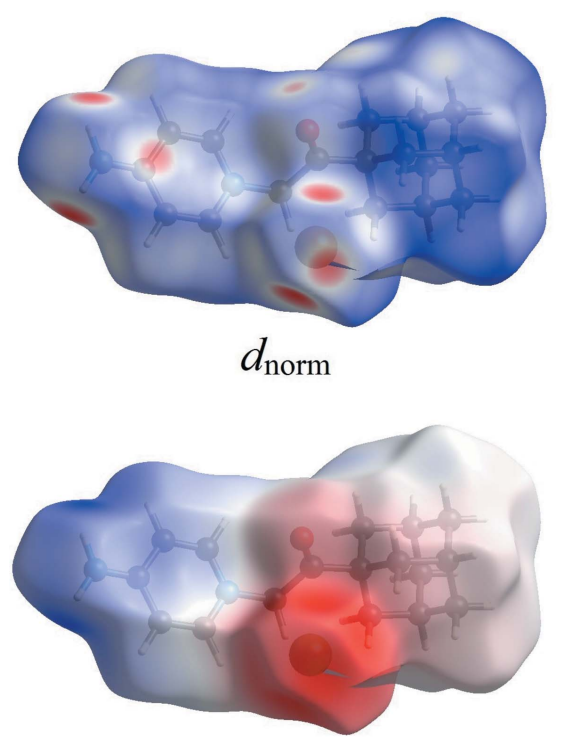

Electrostatic Potential

Figure 3

Hirshfeld surfaces mapped over $d_{\text {norm }}$ and electrostatic potential to visualize the intermolecular contacts in the title salt. The molecule in the ball-and-stick model is in the same orientation shown in the Hirshfeld surface and electrostatic potential plots.

Dark-red spots that are close to atoms H1N2, H2N2, H12A and $\mathrm{Br} 1$ in the $d_{\text {norm }}$ surface mapping are the result of the $\mathrm{N} 2-\mathrm{H} 1 N 2 \cdots \mathrm{Br} 1, \mathrm{~N} 2-\mathrm{H} 2 \mathrm{~N} 2 \cdots \mathrm{Br} 1$ and $\mathrm{C} 12-\mathrm{H} 12 A \cdots \mathrm{Br} 1$ hydrogen bonds (Fig. 4a). This observation is further confirmed by the respective electrostatic potential maps where Br1 shows negative electrostatic potential as a hydrogen
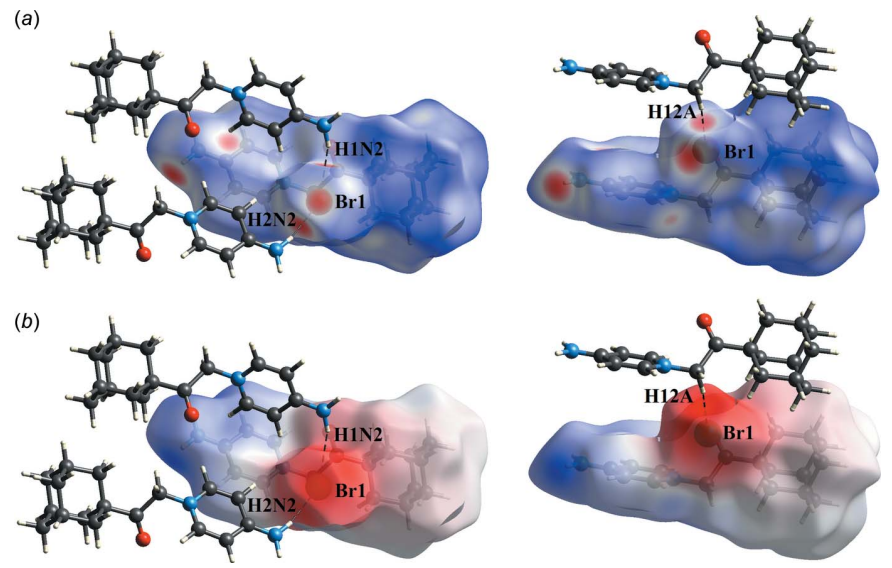

Figure 4

A visualization of the $\mathrm{N}-\mathrm{H} \cdots \mathrm{Br}$ and $\mathrm{C}-\mathrm{H} \cdots \mathrm{Br}$ interactions. (a) $d_{\text {norm }}$ and $(b)$ electrostatic potential mapped on Hirshfeld surfaces in order to visualize the $\mathrm{N}-\mathrm{H} \cdots \mathrm{Br}$ and $\mathrm{C}-\mathrm{H} \cdots \mathrm{Br}$ interactions (black dotted lines). (a)

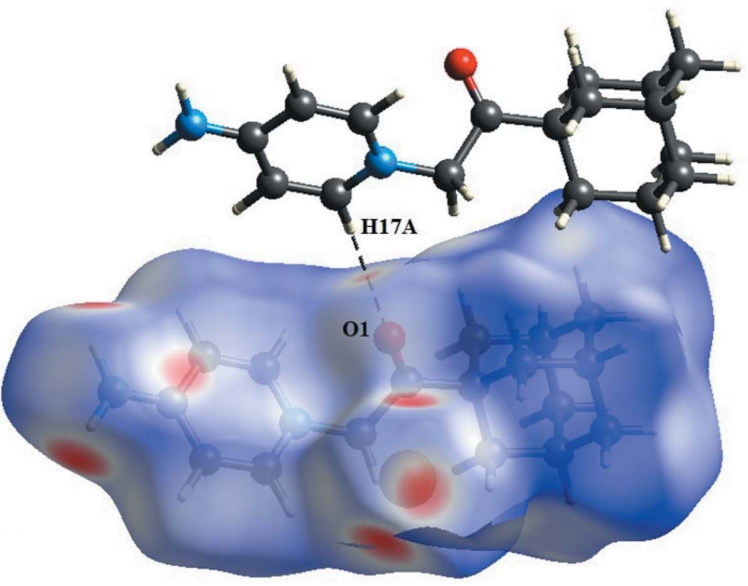

(b)

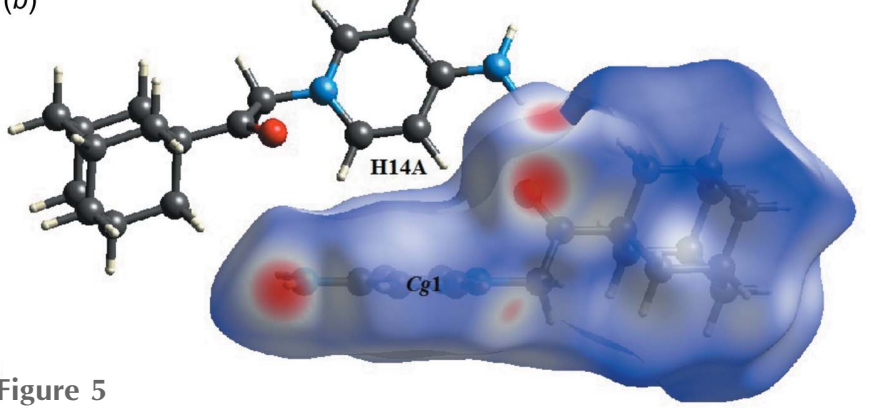

Figure 5

$d_{\text {norm }}$ mapped on Hirshfeld surfaces in order to visualize $(a)$ the $\mathrm{C}-$ $\mathrm{H} \cdots \mathrm{Br}$ hydrogen bond (black dashed line) and $(b)$ the $\mathrm{C}-\mathrm{H} \cdots \pi$ interactions.

acceptor (red region, Fig. $4 b$ ). Beside those two short intermolecular contacts, the $\mathrm{C}-\mathrm{H} \cdots \mathrm{O}$ and $\mathrm{C}-\mathrm{H} \cdots \pi$ interactions are shown as light-red spots on the $d_{\text {norm }}$ surface (Fig. 5).

A quantitative analysis of the intermolecular interactions can be made by studying the fingerprint plots (FP); characteristic pseudo-symmetry wings in the $d_{\mathrm{e}}$ and $d_{\mathrm{i}}$ diagonal axes can be seen in the overall two-dimensional FP (Fig. 6). The most significant intermolecular interactions are the $\mathrm{H} \cdots \mathrm{H}$ interactions $(63.5 \%)$, which appear in the central region of the FP with $d_{\mathrm{e}}=d_{\mathrm{i}} \simeq 2.2 \AA$ (Fig. $6 b$ ). The reciprocal $\mathrm{H} \cdots \mathrm{Br} /$ $\mathrm{Br} \cdots \mathrm{H}$ and $\mathrm{H} \cdots \mathrm{O} / \mathrm{O} \cdots \mathrm{H}$ interactions with $15.9 \%$ and $7.6 \%$ contributions, respectively are present as sharp symmetrical spikes at $d_{\mathrm{e}}+d_{\mathrm{i}} \simeq 2.4$ and $2.5 \AA$, respectively (Fig. $6 c$ and $6 e$ ). The reciprocal $\mathrm{H} \cdots \mathrm{C} / \mathrm{C} \cdots \mathrm{H}$ interactions appear as two symmetrical narrow wings at $d_{\mathrm{e}}+d_{\mathrm{i}} \simeq 2.5 \AA$ and contribute $7.8 \%$ to the Hirshfeld surface (Fig. $6 d$ ). The reciprocal N $\cdots H /$ $\mathrm{H} \cdots \mathrm{N}$ interactions appear as a symmetrical V-shaped wing in the FP map with $d_{\mathrm{e}}+d_{\mathrm{i}} \simeq 2.7 \AA$ and contribute $2.7 \%$ to the Hirshfeld surface (Fig. $6 f$ ). The percentage contributions for other intermolecular contacts are less than $2.6 \%$.

\section{Synthesis and crystallization}

A mixture of 1-adamantly bromomethyl ketone $(2.75 \mathrm{~g}$, $10 \mathrm{mmol})$ and 4-aminopyridine $(0.11 \mathrm{~g}, 1 \mathrm{mmol})$ was dissolved in $10 \mathrm{ml}$ of toluene at room temperature, followed by stirring at $358 \mathrm{~K}$ for $18 \mathrm{~h}$. The completion of the reaction was marked by the amount of the separated solid from the initially clear 
(a)
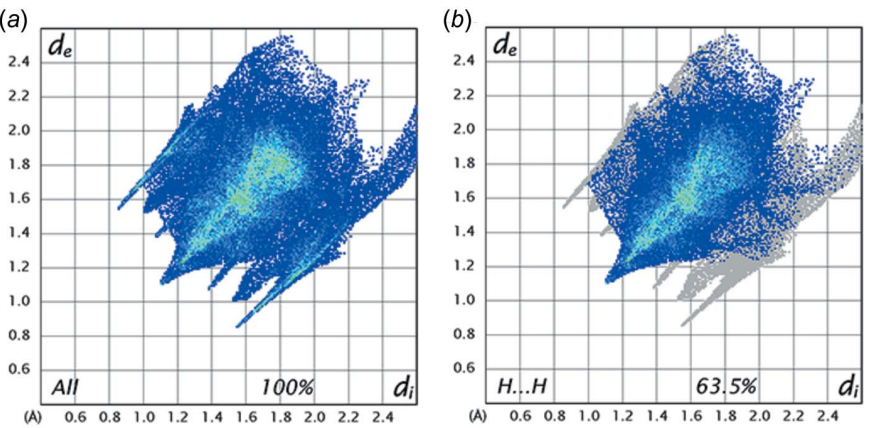

(c)

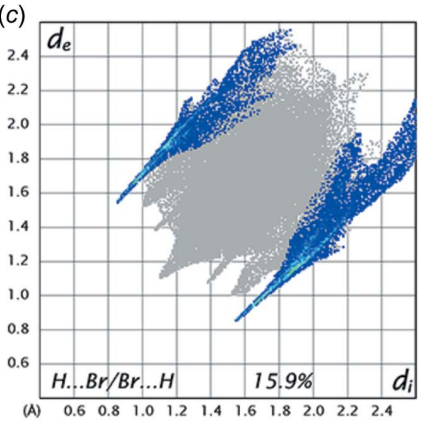

(e)

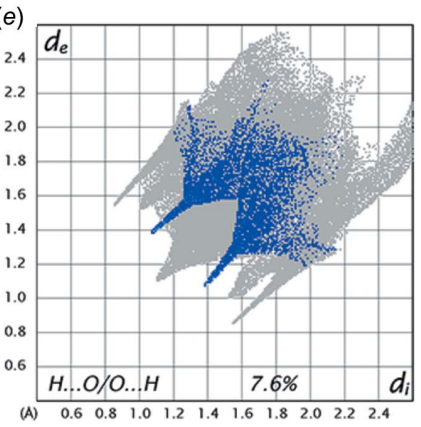

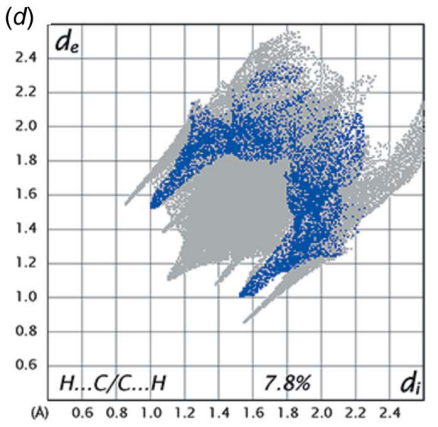

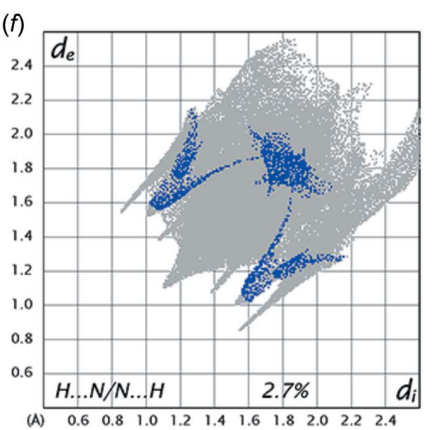

Figure 6

Fingerprint plots.

and homogeneous mixture of the starting materials. The solid was filtered and washed by ethyl acetate. The final pyridiniminium salt was obtained after the solid had been dried under reduced pressure to remove all volatile organic compounds (Said et al., 2017; Sheshadri et al., 2018). Plate-like colourless crystals were obtained by slow evaporation of an acetone solution.

\section{Refinement}

Crystal data, data collection and structure refinement details are summarized in Table 2. C-bound $\mathrm{H}$ atoms were positioned geometrically $[\mathrm{C}-\mathrm{H}=0.93-0.98 \AA]$ and refined using a riding model with $U_{\text {iso }}(\mathrm{H})=1.2 U_{\text {eq }}(\mathrm{C})$. The $\mathrm{N}$-bound $\mathrm{H}$ atoms were located in a difference-Fourier map and freely refined. One outlier (100) was omitted in the last cycles of refinement.

\section{Funding information}

HCK thanks the Malaysian Government for a MyBrain15 scholarship.

Table 2

Experimental details.

Crystal data

Chemical formula

$M_{\mathrm{r}}$

Crystal system, space group

Temperature (K)

$a, b, c(\AA)$

$\beta\left({ }^{\circ}\right)$

$V\left(\AA^{3}\right)$

$Z$

Radiation type

$\mu\left(\mathrm{mm}^{-1}\right)$

Crystal size (mm)

$\mathrm{C}_{17} \mathrm{H}_{23} \mathrm{~N}_{2} \mathrm{O}^{+} \cdot \mathrm{Br}^{-}$

351.28

Monoclinic, $P 2_{1} / c$

294

18.758 (2), 7.1508 (8), 11.9909 (14)

$98.2117(17)$

1591.9 (3)

Mo $K \alpha$

2.58

Data collection

Diffractometer

Absorption correction

$T_{\min }, T_{\max }$

No. of measured, independent and observed $[I>2 \sigma(I)]$ reflections

$R_{\text {int }}$

$(\sin \theta / \lambda)_{\max }\left(\AA^{-1}\right)$

$0.38 \times 0.25 \times 0.09$

Refinement

$R\left[F^{2}>2 \sigma\left(F^{2}\right)\right], w R\left(F^{2}\right), S$

No. of reflections

No. of parameters

$\mathrm{H}$-atom treatment

$\Delta \rho_{\max }, \Delta \rho_{\min }\left(\mathrm{e} \AA^{-3}\right)$

Bruker APEXII DUO CCD areadetector

Multi-scan (SADABS; Bruker, 2012)

$0.320,0.408$

$35418,4897,3392$

0.051

0.716

$0.035,0.080,1.01$

4897

198

$\mathrm{H}$ atoms treated by a mixture of independent and constrained refinement

$0.39,-0.25$

Computer programs: APEX2 and SAINT (Bruker, 2012), SHELXS97 (Sheldrick, 2008), SHELXL2013 (Sheldrick, 2015), Mercury (Macrae et al., 2006) and PLATON (Spek, 2009).

\section{References}

Allen, F. H. (2002). Acta Cryst. B58, 380-388.

Ashcroft, F. M. (1999). Ion channels and disease. New York: Academic Press.

Ballester, P. (2008). Recognition of anions. New York: Springer Science \& Business Media.

Blanchard, L. A., Gu, Z. \& Brennecke, J. F. (2001). J. Phys. Chem. B, 105, 2437-2444.

Bruker (2012). APEX2, SAINT and SADABS. Bruker AXS Inc., Madison, Wisconsin, USA.

Chauvin, Y. \& Olivier-Bourbigou, H. (1995). Chemtech, 25, 26-30.

Chidan Kumar, C. S., Sim, A. J., Ng, W. Z., Chia, T. S., Loh, W.-S., Kwong, H. C., Quah, C. K., Naveen, S., Lokanath, N. K. \& Warad, I. (2017). Acta Cryst. E73, 927-931.

Crosthwaite, J. M., Aki, S. N., Maginn, E. J. \& Brennecke, J. F. (2004). J. Phys. Chem. B, 108, 5113-5119.

Davis, J. H. Jr (2004). Chem. Lett. 33, 1072-1077.

Frontera, A., Gamez, P., Mascal, M., Mooibroek, T. J. \& Reedijk, J. (2011). Angew. Chem. 123, 9736-9756.

Hay, B. P. \& Bryantsev, V. S. (2008). Chem. Commun. pp. 2417-2428.

Holbrey, J. \& Seddon, K. (1999). Clean Prod. Process. 1, 223-236.

Lamanna, G., Russier, J., Dumortier, H. \& Bianco, A. (2012). Biomaterials, 33, 5610-5617.

Liu, J., Obando, D., Liao, V., Lifa, T. \& Codd, R. (2011). Eur. J. Med. Chem. 46, 1949-1963.

Macrae, C. F., Edgington, P. R., McCabe, P., Pidcock, E., Shields, G. P., Taylor, R., Towler, M. \& van de Streek, J. (2006). J. Appl. Cryst. 39, 453-457.

Piérard, G. E., Piérard-Franchimont, C., Paquet, P. \& Quatresooz, P. (2009). Expert Opin. Drug Metab. Toxicol. 5, 1565-1575. 
Priyanka, B., Anitha, K., Shirisha, S., Dipankar, B. \& Rajesh, K. (2013). Int. Res. J. Pharm. App. Sci. 3, 93-101.

Reisberg, B., Doody, R., Stöffler, A., Schmitt, F., Ferris, S., \& Möbius, H. J. (2003). N. Engl. J. Med. 348, 1333-1341.

Rouchal, M., Nečas, M. \& Vícha, R. (2011). Acta Cryst. E67, o3198.

Said, M. A., Aouad, M. R., Hughes, D. L., Almehmadi, M. A. \& Messali, M. (2017). Acta Cryst. E73, 1831-1834.

Schottel, B. L., Chifotides, H. T. \& Dunbar, K. R. (2008). Chem. Soc. Rev. 37, 68-83.

Sharmila, N., Sundar, T. V., Yasodha, A., Puratchikody, A. \& Sridhar, B. (2014). Acta Cryst. E70, o1293-01294.

Sheldrick, G. M. (2008). Acta Cryst. A64, 112-122.

Sheldrick, G. M. (2015). Acta Cryst. C71, 3-8.

Sheshadri, S. N., Kwong, H. C., Chidan Kumar, C. S., Quah, C. K., Siddaraju, B. P., Veeraiah, M. K., Hamid, M. A. B. A. \& Warad, I. (2018). Acta Cryst. E74, 752-756.
Spackman, M. A. \& Jayatilaka, D. (2009). CrystEngComm, 11, 19-32. Spackman, M. A. \& McKinnon, J. J. (2002). CrystEngComm, 4, 378392.

Spackman, M. A., McKinnon, J. J. \& Jayatilaka, D. (2008). CrystEngComm, 10, 377-388.

Spek, A. L. (2009). Acta Cryst. D65, 148-155.

Wolff, S. K., Grimwood, D. J., McKinnon, J. J., Turner, M. J., Jayatilaka, D. \& Spackman, M. A. (2012). CrystalExplorer. University of Western Australia.

Yue, W. W., Li, H. J., Xiang, T., Qin, H., Sun, S. D. \& Zhao, C. S. (2013). J. Membr. Sci. 446, 79-91.

Zettl, H., Schubert-Zsilavecz, M. \& Steinhilber, D. (2010). ChemMedChem, 5, 179-185.

Zoidis, G., Kolocouris, N., Kelly, J. M., Prathalingam, S. R., Naesens, L. \& De Clercq, E. (2010). Eur. J. Med. Chem. 45, 5022-5030. 


\section{supporting information}

Acta Cryst. (2018). E74, 1030-1034 [https://doi.org/10.1107/S2056989018009131]

Crystal structure and Hirshfeld surface analysis of a pyridiniminium bromide salt: 1-[2-(adamantan-1-yl)-2-oxoethyl]pyridin-4-iminium bromide

Huey Chong Kwong, Imdad Mahmud Pathi, C. S. Chidan Kumar, Ching Kheng Quah and Md. Azharul Arafath

Computing details

Data collection: APEX2 (Bruker, 2012); cell refinement: SAINT (Bruker, 2012); data reduction: SAINT (Bruker, 2012); program(s) used to solve structure: SHELXS97 (Sheldrick, 2008); program(s) used to refine structure: SHELXL2013 (Sheldrick, 2015); molecular graphics: SHELXL2013 (Sheldrick, 2015) and Mercury (Macrae et al., 2006); software used to prepare material for publication: SHELXL2013 (Sheldrick, 2015) and PLATON (Spek, 2009).

1-[2-(Adamantan-1-yl)-2-oxoethyl]pyridin-4-iminium bromide

Crystal data

$\mathrm{C}_{17} \mathrm{H}_{23} \mathrm{~N}_{2} \mathrm{O}^{+} \cdot \mathrm{Br}^{-}$

$M_{r}=351.28$

Monoclinic, $P 2_{1} / c$

$a=18.758(2) \AA$

$b=7.1508(8) \AA$

$c=11.9909$ (14) $\AA$

$\beta=98.2117(17)^{\circ}$

$V=1591.9(3) \AA^{3}$

$Z=4$

\section{Data collection}

Bruker APEXII DUO CCD area-detector diffractometer

Radiation source: fine-focus sealed tube Graphite monochromator $\varphi$ and $\omega$ scans

Absorption correction: multi-scan

(SADABS; Bruker, 2012)

$T_{\min }=0.320, T_{\max }=0.408$

\section{Refinement}

Refinement on $F^{2}$

Least-squares matrix: full

$R\left[F^{2}>2 \sigma\left(F^{2}\right)\right]=0.035$

$w R\left(F^{2}\right)=0.080$

$S=1.01$

4897 reflections

198 parameters

0 restraints
$F(000)=728$

$D_{\mathrm{x}}=1.466 \mathrm{Mg} \mathrm{m}^{-3}$

Mo $K \alpha$ radiation, $\lambda=0.71073 \AA$

Cell parameters from 7278 reflections

$\theta=3.1-25.2^{\circ}$

$\mu=2.58 \mathrm{~mm}^{-1}$

$T=294 \mathrm{~K}$

Plate, colourless

$0.38 \times 0.25 \times 0.09 \mathrm{~mm}$

35418 measured reflections

4897 independent reflections

3392 reflections with $I>2 \sigma(I)$

$R_{\text {int }}=0.051$

$\theta_{\text {max }}=30.6^{\circ}, \theta_{\min }=2.2^{\circ}$

$h=-26 \rightarrow 26$

$k=-10 \rightarrow 10$

$l=-17 \rightarrow 17$

Hydrogen site location: mixed

$\mathrm{H}$ atoms treated by a mixture of independent and constrained refinement

$w=1 /\left[\sigma^{2}\left(F_{\mathrm{o}}{ }^{2}\right)+(0.0364 P)^{2}+0.3328 P\right]$

where $P=\left(F_{\mathrm{o}}^{2}+2 F_{\mathrm{c}}^{2}\right) / 3$

$(\Delta / \sigma)_{\max }=0.001$

$\Delta \rho_{\max }=0.39 \mathrm{e} \AA^{-3}$

$\Delta \rho_{\min }=-0.25$ e $\AA^{-3}$ 


\section{Special details}

Experimental. The following wavelength and cell were deduced by SADABS from the direction cosines etc. They are given here for emergency use only: CELL 0.7107812 .0097 .16218 .79989 .98398 .20290 .025

Geometry. All esds (except the esd in the dihedral angle between two 1.s. planes) are estimated using the full covariance matrix. The cell esds are taken into account individually in the estimation of esds in distances, angles and torsion angles; correlations between esds in cell parameters are only used when they are defined by crystal symmetry. An approximate (isotropic) treatment of cell esds is used for estimating esds involving l.s. planes.

Fractional atomic coordinates and isotropic or equivalent isotropic displacement parameters $\left(\AA^{2}\right)$

\begin{tabular}{|c|c|c|c|c|}
\hline & $x$ & $y$ & $z$ & $U_{\text {iso }} * / U_{\text {eq }}$ \\
\hline $\mathrm{Br} 1$ & $0.18668(2)$ & $0.58832(3)$ & $0.43377(2)$ & $0.04161(8)$ \\
\hline $\mathrm{O} 1$ & $0.16460(7)$ & $0.2172(2)$ & $0.22373(11)$ & $0.0449(3)$ \\
\hline N1 & $0.09073(8)$ & 0.11925 (19) & $0.39566(12)$ & $0.0287(3)$ \\
\hline $\mathrm{N} 2$ & $-0.12926(9)$ & $0.1691(3)$ & $0.35275(16)$ & $0.0399(4)$ \\
\hline H1N2 & $-0.1522(14)$ & $0.122(3)$ & $0.294(2)$ & $0.060(8)^{*}$ \\
\hline $\mathrm{H} 2 \mathrm{~N} 2$ & $-0.1491(11)$ & $0.232(3)$ & $0.3994(18)$ & $0.046(6)^{*}$ \\
\hline $\mathrm{C} 1$ & $0.28202(10)$ & $0.1231(3)$ & $0.31057(14)$ & $0.0334(4)$ \\
\hline $\mathrm{C} 2$ & $0.32236(11)$ & $0.1050(4)$ & $0.43067(16)$ & $0.0487(6)$ \\
\hline $\mathrm{H} 2 \mathrm{~A}$ & 0.3014 & 0.0049 & 0.4699 & $0.058^{*}$ \\
\hline $\mathrm{H} 2 \mathrm{~B}$ & 0.3180 & 0.2204 & 0.4717 & $0.058^{*}$ \\
\hline $\mathrm{C} 3$ & $0.40229(11)$ & $0.0632(4)$ & $0.4260(2)$ & $0.0617(7)$ \\
\hline $\mathrm{H} 3 \mathrm{~A}$ & 0.4278 & 0.0525 & 0.5029 & $0.074 *$ \\
\hline $\mathrm{C} 4$ & $0.40873(13)$ & $-0.1190(4)$ & $0.3647(2)$ & $0.0647(7)$ \\
\hline $\mathrm{H} 4 \mathrm{~A}$ & 0.3876 & -0.2193 & 0.4034 & $0.078^{*}$ \\
\hline $\mathrm{H} 4 \mathrm{~B}$ & 0.4591 & -0.1483 & 0.3636 & $0.078 *$ \\
\hline $\mathrm{C} 5$ & $0.37011(12)$ & $-0.1023(4)$ & $0.2448(2)$ & $0.0551(6)$ \\
\hline $\mathrm{H} 5 \mathrm{~A}$ & 0.3751 & -0.2198 & 0.2045 & $0.066^{*}$ \\
\hline C6 & $0.28984(11)$ & $-0.0621(3)$ & $0.24763(17)$ & $0.0433(5)$ \\
\hline H6A & 0.2687 & -0.1634 & 0.2855 & $0.052^{*}$ \\
\hline H6B & 0.2646 & -0.0533 & 0.1713 & $0.052 *$ \\
\hline $\mathrm{C} 7$ & $0.31564(11)$ & $0.2816(3)$ & $0.2499(2)$ & $0.0513(5)$ \\
\hline H7A & 0.3111 & 0.3987 & 0.2891 & $0.062 *$ \\
\hline H7B & 0.2906 & 0.2941 & 0.1737 & $0.062 *$ \\
\hline $\mathrm{C} 8$ & $0.43485(13)$ & $0.2227(5)$ & $0.3659(3)$ & $0.0765(8)$ \\
\hline H8A & 0.4306 & 0.3390 & 0.4060 & $0.092^{*}$ \\
\hline H8B & 0.4856 & 0.1988 & 0.3639 & $0.092 *$ \\
\hline C9 & $0.40260(13)$ & $0.0572(4)$ & $0.1841(2)$ & $0.0672(8)$ \\
\hline H9A & 0.4531 & 0.0318 & 0.1808 & $0.081^{*}$ \\
\hline H9B & 0.3779 & 0.0676 & 0.1076 & $0.081^{*}$ \\
\hline $\mathrm{C} 10$ & $0.39530(12)$ & $0.2385(4)$ & $0.2467(2)$ & $0.0653(7)$ \\
\hline $\mathrm{H} 10 \mathrm{~A}$ & 0.4166 & 0.3403 & 0.2077 & $0.078^{*}$ \\
\hline C11 & $0.20180(10)$ & $0.1573(3)$ & $0.30656(14)$ & $0.0317(4)$ \\
\hline $\mathrm{C} 12$ & $0.16924(9)$ & $0.0976(3)$ & $0.40968(15)$ & $0.0301(4)$ \\
\hline $\mathrm{H} 12 \mathrm{~A}$ & 0.1813 & -0.0324 & 0.4260 & $0.036^{*}$ \\
\hline H12B & 0.1905 & 0.1715 & 0.4738 & $0.036^{*}$ \\
\hline $\mathrm{C} 13$ & $0.04945(10)$ & $0.0291(3)$ & $0.31040(15)$ & $0.0357(4)$ \\
\hline $\mathrm{H} 13 \mathrm{~A}$ & 0.0718 & -0.0446 & 0.2616 & $0.043^{*}$ \\
\hline
\end{tabular}


supporting information

$\begin{array}{lllll}\text { C14 } & -0.02299(10) & 0.0429(3) & 0.29395(15) & 0.0353(4) \\ \text { H14A } & -0.0498 & -0.0198 & 0.2341 & 0.042^{*} \\ \text { C15 } & -0.05818(10) & 0.1526(3) & 0.36771(14) & 0.0295(4) \\ \text { C16 } & -0.01401(9) & 0.2433(2) & 0.45605(14) & 0.0300(4) \\ \text { H16A } & -0.0348 & 0.3164 & 0.5069 & 0.036^{*} \\ \text { C17 } & 0.05879(10) & 0.2251(2) & 0.46772(14) & 0.0297(4) \\ \text { H17A } & 0.0872 & 0.2867 & 0.5264 & 0.036^{*}\end{array}$

Atomic displacement parameters $\left(\AA^{2}\right)$

\begin{tabular}{lllllll}
\hline & $U^{11}$ & $U^{22}$ & $U^{33}$ & $U^{12}$ & $U^{13}$ & $U^{23}$ \\
\hline Br1 & $0.05492(14)$ & $0.03289(11)$ & $0.03750(11)$ & $0.00147(9)$ & $0.00823(8)$ & $-0.00127(8)$ \\
O1 & $0.0371(8)$ & $0.0609(10)$ & $0.0352(7)$ & $0.0074(7)$ & $0.0001(6)$ & $0.0094(7)$ \\
N1 & $0.0278(8)$ & $0.0256(8)$ & $0.0330(7)$ & $-0.0007(6)$ & $0.0051(6)$ & $-0.0020(6)$ \\
N2 & $0.0300(9)$ & $0.0507(11)$ & $0.0389(9)$ & $0.0022(8)$ & $0.0051(8)$ & $-0.0078(8)$ \\
C1 & $0.0276(9)$ & $0.0426(11)$ & $0.0295(8)$ & $-0.0005(8)$ & $0.0028(7)$ & $0.0024(7)$ \\
C2 & $0.0304(10)$ & $0.0816(17)$ & $0.0330(9)$ & $-0.0033(11)$ & $0.0009(8)$ & $-0.0015(10)$ \\
C3 & $0.0273(11)$ & $0.109(2)$ & $0.0454(12)$ & $0.0014(13)$ & $-0.0049(9)$ & $0.0035(13)$ \\
C4 & $0.0344(12)$ & $0.092(2)$ & $0.0681(16)$ & $0.0187(13)$ & $0.0077(11)$ & $0.0252(14)$ \\
C5 & $0.0452(13)$ & $0.0659(16)$ & $0.0550(13)$ & $0.0171(11)$ & $0.0094(10)$ & $-0.0039(11)$ \\
C6 & $0.0355(11)$ & $0.0506(13)$ & $0.0427(10)$ & $0.0056(9)$ & $0.0018(8)$ & $-0.0041(9)$ \\
C7 & $0.0434(12)$ & $0.0528(14)$ & $0.0613(13)$ & $0.0005(10)$ & $0.0198(10)$ & $0.0138(11)$ \\
C8 & $0.0323(13)$ & $0.102(2)$ & $0.096(2)$ & $-0.0162(14)$ & $0.0110(13)$ & $-0.0112(18)$ \\
C9 & $0.0450(14)$ & $0.105(2)$ & $0.0562(14)$ & $0.0200(14)$ & $0.0234(11)$ & $0.0144(14)$ \\
C10 & $0.0453(14)$ & $0.0729(18)$ & $0.0839(18)$ & $-0.0032(12)$ & $0.0307(13)$ & $0.0217(15)$ \\
C11 & $0.0312(10)$ & $0.0329(9)$ & $0.0309(9)$ & $-0.0004(8)$ & $0.0035(7)$ & $-0.0012(7)$ \\
C12 & $0.0248(9)$ & $0.0298(9)$ & $0.0357(8)$ & $0.0041(7)$ & $0.0042(7)$ & $0.0028(7)$ \\
C13 & $0.0352(10)$ & $0.0347(10)$ & $0.0380(9)$ & $-0.0021(8)$ & $0.0076(8)$ & $-0.0130(8)$ \\
C14 & $0.0345(10)$ & $0.0373(10)$ & $0.0342(9)$ & $-0.0045(8)$ & $0.0052(8)$ & $-0.0107(8)$ \\
C15 & $0.0307(10)$ & $0.0277(8)$ & $0.0308(8)$ & $0.0011(7)$ & $0.0067(7)$ & $0.0031(7)$ \\
C16 & $0.0336(10)$ & $0.0288(9)$ & $0.0290(8)$ & $0.0024(8)$ & $0.0089(7)$ & $-0.0017(7)$ \\
C17 & $0.0362(10)$ & $0.0256(8)$ & $0.0275(8)$ & $-0.0022(7)$ & $0.0053(7)$ & $-0.0013(7)$ \\
& & & & & &
\end{tabular}

Geometric parameters ( $\left.\AA,{ }^{\circ}\right)$

\begin{tabular}{llll}
\hline $\mathrm{O} 1-\mathrm{C} 11$ & $1.208(2)$ & $\mathrm{C} 6-\mathrm{H} 6 \mathrm{~A}$ & 0.9700 \\
$\mathrm{~N} 1-\mathrm{C} 17$ & $1.352(2)$ & $\mathrm{C} 6-\mathrm{H} 6 \mathrm{~B}$ & 0.9700 \\
$\mathrm{~N} 1-\mathrm{C} 13$ & $1.354(2)$ & $\mathrm{C} 7-\mathrm{C} 10$ & $1.531(3)$ \\
$\mathrm{N} 1-\mathrm{C} 12$ & $1.466(2)$ & $\mathrm{C} 7-\mathrm{H} 7 \mathrm{~A}$ & 0.9700 \\
$\mathrm{~N} 2-\mathrm{C} 15$ & $1.325(2)$ & $\mathrm{C} 7-\mathrm{H} 7 \mathrm{~B}$ & 0.9700 \\
$\mathrm{~N} 2-\mathrm{H} 1 \mathrm{~N} 2$ & $0.85(3)$ & $\mathrm{C} 8-\mathrm{C} 10$ & $1.517(4)$ \\
$\mathrm{N} 2-\mathrm{H} 2 \mathrm{~N} 2$ & $0.84(2)$ & $\mathrm{C} 8-\mathrm{H} 8 \mathrm{~A}$ & 0.9700 \\
$\mathrm{C} 1-\mathrm{C} 11$ & $1.518(3)$ & $\mathrm{C} 8-\mathrm{H} 8 \mathrm{~B}$ & 0.9700 \\
$\mathrm{C} 1-\mathrm{C} 7$ & $1.530(3)$ & $\mathrm{C} 9-\mathrm{C} 10$ & $1.514(4)$ \\
$\mathrm{C} 1-\mathrm{C} 2$ & $1.534(3)$ & $\mathrm{C} 9-\mathrm{H} 9 \mathrm{~A}$ & 0.9700 \\
$\mathrm{C} 1-\mathrm{C} 6$ & $1.542(3)$ & $\mathrm{C} 9-\mathrm{H} 9 \mathrm{~B}$ & 0.9700 \\
$\mathrm{C} 2-\mathrm{C} 3$ & $1.538(3)$ & $\mathrm{C} 10-\mathrm{H} 10 \mathrm{~A}$ & 0.9800 \\
$\mathrm{C} 2-\mathrm{H} 2 \mathrm{~A}$ & 0.9700 & $\mathrm{C} 11-\mathrm{C} 12$ & $1.517(2)$
\end{tabular}




\begin{tabular}{|c|c|c|c|}
\hline $\mathrm{C} 2-\mathrm{H} 2 \mathrm{~B}$ & 0.9700 & $\mathrm{C} 12-\mathrm{H} 12 \mathrm{~A}$ & 0.9700 \\
\hline $\mathrm{C} 3-\mathrm{C} 4$ & $1.509(4)$ & $\mathrm{C} 12-\mathrm{H} 12 \mathrm{~B}$ & 0.9700 \\
\hline $\mathrm{C} 3-\mathrm{C} 8$ & $1.523(4)$ & $\mathrm{C} 13-\mathrm{C} 14$ & $1.348(3)$ \\
\hline $\mathrm{C} 3-\mathrm{H} 3 \mathrm{~A}$ & 0.9800 & $\mathrm{C} 13-\mathrm{H} 13 \mathrm{~A}$ & 0.9300 \\
\hline $\mathrm{C} 4-\mathrm{C} 5$ & $1.520(3)$ & $\mathrm{C} 14-\mathrm{C} 15$ & $1.414(2)$ \\
\hline $\mathrm{C} 4-\mathrm{H} 4 \mathrm{~A}$ & 0.9700 & $\mathrm{C} 14-\mathrm{H} 14 \mathrm{~A}$ & 0.9300 \\
\hline $\mathrm{C} 4-\mathrm{H} 4 \mathrm{~B}$ & 0.9700 & $\mathrm{C} 15-\mathrm{C} 16$ & $1.407(2)$ \\
\hline $\mathrm{C} 5-\mathrm{C} 9$ & $1.526(4)$ & $\mathrm{C} 16-\mathrm{C} 17$ & $1.359(2)$ \\
\hline $\mathrm{C} 5-\mathrm{C} 6$ & $1.538(3)$ & C16-H16A & 0.9300 \\
\hline $\mathrm{C} 5-\mathrm{H} 5 \mathrm{~A}$ & 0.9800 & C17-H17A & 0.9300 \\
\hline $\mathrm{C} 17-\mathrm{N} 1-\mathrm{C} 13$ & $119.43(15)$ & $\mathrm{C} 1-\mathrm{C} 7-\mathrm{H} 7 \mathrm{~B}$ & 109.8 \\
\hline $\mathrm{C} 17-\mathrm{N} 1-\mathrm{C} 12$ & $121.03(15)$ & $\mathrm{C} 10-\mathrm{C} 7-\mathrm{H} 7 \mathrm{~B}$ & 109.8 \\
\hline $\mathrm{C} 13-\mathrm{N} 1-\mathrm{C} 12$ & $119.53(15)$ & $\mathrm{H} 7 \mathrm{~A}-\mathrm{C} 7-\mathrm{H} 7 \mathrm{~B}$ & 108.2 \\
\hline $\mathrm{C} 15-\mathrm{N} 2-\mathrm{H} 1 \mathrm{~N} 2$ & $117.3(18)$ & $\mathrm{C} 10-\mathrm{C} 8-\mathrm{C} 3$ & $109.1(2)$ \\
\hline $\mathrm{C} 15-\mathrm{N} 2-\mathrm{H} 2 \mathrm{~N} 2$ & $119.2(14)$ & $\mathrm{C} 10-\mathrm{C} 8-\mathrm{H} 8 \mathrm{~A}$ & 109.9 \\
\hline $\mathrm{H} 1 \mathrm{~N} 2-\mathrm{N} 2-\mathrm{H} 2 \mathrm{~N} 2$ & $123(2)$ & $\mathrm{C} 3-\mathrm{C} 8-\mathrm{H} 8 \mathrm{~A}$ & 109.9 \\
\hline $\mathrm{C} 11-\mathrm{C} 1-\mathrm{C} 7$ & $109.85(16)$ & $\mathrm{C} 10-\mathrm{C} 8-\mathrm{H} 8 \mathrm{~B}$ & 109.9 \\
\hline $\mathrm{C} 11-\mathrm{C} 1-\mathrm{C} 2$ & $113.40(15)$ & $\mathrm{C} 3-\mathrm{C} 8-\mathrm{H} 8 \mathrm{~B}$ & 109.9 \\
\hline $\mathrm{C} 7-\mathrm{C} 1-\mathrm{C} 2$ & $109.10(17)$ & $\mathrm{H} 8 \mathrm{~A}-\mathrm{C} 8-\mathrm{H} 8 \mathrm{~B}$ & 108.3 \\
\hline $\mathrm{C} 11-\mathrm{C} 1-\mathrm{C} 6$ & $106.61(15)$ & $\mathrm{C} 10-\mathrm{C} 9-\mathrm{C} 5$ & $109.47(19)$ \\
\hline $\mathrm{C} 7-\mathrm{C} 1-\mathrm{C} 6$ & $109.20(16)$ & $\mathrm{C} 10-\mathrm{C} 9-\mathrm{H} 9 \mathrm{~A}$ & 109.8 \\
\hline $\mathrm{C} 2-\mathrm{C} 1-\mathrm{C} 6$ & $108.58(17)$ & $\mathrm{C} 5-\mathrm{C} 9-\mathrm{H} 9 \mathrm{~A}$ & 109.8 \\
\hline $\mathrm{C} 1-\mathrm{C} 2-\mathrm{C} 3$ & $109.56(16)$ & $\mathrm{C} 10-\mathrm{C} 9-\mathrm{H} 9 \mathrm{~B}$ & 109.8 \\
\hline $\mathrm{C} 1-\mathrm{C} 2-\mathrm{H} 2 \mathrm{~A}$ & 109.8 & $\mathrm{C} 5-\mathrm{C} 9-\mathrm{H} 9 \mathrm{~B}$ & 109.8 \\
\hline $\mathrm{C} 3-\mathrm{C} 2-\mathrm{H} 2 \mathrm{~A}$ & 109.8 & $\mathrm{H} 9 \mathrm{~A}-\mathrm{C} 9-\mathrm{H} 9 \mathrm{~B}$ & 108.2 \\
\hline $\mathrm{C} 1-\mathrm{C} 2-\mathrm{H} 2 \mathrm{~B}$ & 109.8 & $\mathrm{C} 9-\mathrm{C} 10-\mathrm{C} 8$ & $109.7(2)$ \\
\hline $\mathrm{C} 3-\mathrm{C} 2-\mathrm{H} 2 \mathrm{~B}$ & 109.8 & $\mathrm{C} 9-\mathrm{C} 10-\mathrm{C} 7$ & $110.0(2)$ \\
\hline $\mathrm{H} 2 \mathrm{~A}-\mathrm{C} 2-\mathrm{H} 2 \mathrm{~B}$ & 108.2 & $\mathrm{C} 8-\mathrm{C} 10-\mathrm{C} 7$ & $109.8(2)$ \\
\hline $\mathrm{C} 4-\mathrm{C} 3-\mathrm{C} 8$ & $110.5(2)$ & $\mathrm{C} 9-\mathrm{C} 10-\mathrm{H} 10 \mathrm{~A}$ & 109.1 \\
\hline $\mathrm{C} 4-\mathrm{C} 3-\mathrm{C} 2$ & $109.4(2)$ & $\mathrm{C} 8-\mathrm{C} 10-\mathrm{H} 10 \mathrm{~A}$ & 109.1 \\
\hline $\mathrm{C} 8-\mathrm{C} 3-\mathrm{C} 2$ & $109.3(2)$ & $\mathrm{C} 7-\mathrm{C} 10-\mathrm{H} 10 \mathrm{~A}$ & 109.1 \\
\hline $\mathrm{C} 4-\mathrm{C} 3-\mathrm{H} 3 \mathrm{~A}$ & 109.2 & $\mathrm{O} 1-\mathrm{C} 11-\mathrm{C} 12$ & $121.17(16)$ \\
\hline $\mathrm{C} 8-\mathrm{C} 3-\mathrm{H} 3 \mathrm{~A}$ & 109.2 & $\mathrm{O} 1-\mathrm{C} 11-\mathrm{C} 1$ & $122.46(16)$ \\
\hline $\mathrm{C} 2-\mathrm{C} 3-\mathrm{H} 3 \mathrm{~A}$ & 109.2 & $\mathrm{C} 12-\mathrm{C} 11-\mathrm{C} 1$ & $116.20(15)$ \\
\hline $\mathrm{C} 3-\mathrm{C} 4-\mathrm{C} 5$ & $109.4(2)$ & $\mathrm{N} 1-\mathrm{C} 12-\mathrm{C} 11$ & $113.06(14)$ \\
\hline $\mathrm{C} 3-\mathrm{C} 4-\mathrm{H} 4 \mathrm{~A}$ & 109.8 & $\mathrm{~N} 1-\mathrm{C} 12-\mathrm{H} 12 \mathrm{~A}$ & 109.0 \\
\hline $\mathrm{C} 5-\mathrm{C} 4-\mathrm{H} 4 \mathrm{~A}$ & 109.8 & $\mathrm{C} 11-\mathrm{C} 12-\mathrm{H} 12 \mathrm{~A}$ & 109.0 \\
\hline $\mathrm{C} 3-\mathrm{C} 4-\mathrm{H} 4 \mathrm{~B}$ & 109.8 & $\mathrm{~N} 1-\mathrm{C} 12-\mathrm{H} 12 \mathrm{~B}$ & 109.0 \\
\hline $\mathrm{C} 5-\mathrm{C} 4-\mathrm{H} 4 \mathrm{~B}$ & 109.8 & $\mathrm{C} 11-\mathrm{C} 12-\mathrm{H} 12 \mathrm{~B}$ & 109.0 \\
\hline $\mathrm{H} 4 \mathrm{~A}-\mathrm{C} 4-\mathrm{H} 4 \mathrm{~B}$ & 108.2 & $\mathrm{H} 12 \mathrm{~A}-\mathrm{C} 12-\mathrm{H} 12 \mathrm{~B}$ & 107.8 \\
\hline $\mathrm{C} 4-\mathrm{C} 5-\mathrm{C} 9$ & $109.9(2)$ & $\mathrm{C} 14-\mathrm{C} 13-\mathrm{N} 1$ & $122.10(16)$ \\
\hline $\mathrm{C} 4-\mathrm{C} 5-\mathrm{C} 6$ & $109.24(19)$ & $\mathrm{C} 14-\mathrm{C} 13-\mathrm{H} 13 \mathrm{~A}$ & 119.0 \\
\hline $\mathrm{C} 9-\mathrm{C} 5-\mathrm{C} 6$ & $109.26(19)$ & $\mathrm{N} 1-\mathrm{C} 13-\mathrm{H} 13 \mathrm{~A}$ & 119.0 \\
\hline $\mathrm{C} 4-\mathrm{C} 5-\mathrm{H} 5 \mathrm{~A}$ & 109.5 & $\mathrm{C} 13-\mathrm{C} 14-\mathrm{C} 15$ & $119.97(17)$ \\
\hline $\mathrm{C} 9-\mathrm{C} 5-\mathrm{H} 5 \mathrm{~A}$ & 109.5 & $\mathrm{C} 13-\mathrm{C} 14-\mathrm{H} 14 \mathrm{~A}$ & 120.0 \\
\hline $\mathrm{C} 6-\mathrm{C} 5-\mathrm{H} 5 \mathrm{~A}$ & 109.5 & $\mathrm{C} 15-\mathrm{C} 14-\mathrm{H} 14 \mathrm{~A}$ & 120.0 \\
\hline $\mathrm{C} 5-\mathrm{C} 6-\mathrm{C} 1$ & $109.47(17)$ & $\mathrm{N} 2-\mathrm{C} 15-\mathrm{C} 16$ & $122.28(17)$ \\
\hline
\end{tabular}




\begin{tabular}{|c|c|c|c|}
\hline $\mathrm{C} 5-\mathrm{C} 6-\mathrm{H} 6 \mathrm{~A}$ & 109.8 & $\mathrm{~N} 2-\mathrm{C} 15-\mathrm{C} 14$ & $121.01(17)$ \\
\hline $\mathrm{C} 1-\mathrm{C} 6-\mathrm{H} 6 \mathrm{~A}$ & 109.8 & $\mathrm{C} 16-\mathrm{C} 15-\mathrm{C} 14$ & $116.71(16)$ \\
\hline $\mathrm{C} 5-\mathrm{C} 6-\mathrm{H} 6 \mathrm{~B}$ & 109.8 & $\mathrm{C} 17-\mathrm{C} 16-\mathrm{C} 15$ & $120.57(16)$ \\
\hline $\mathrm{C} 1-\mathrm{C} 6-\mathrm{H} 6 \mathrm{~B}$ & 109.8 & $\mathrm{C} 17-\mathrm{C} 16-\mathrm{H} 16 \mathrm{~A}$ & 119.7 \\
\hline $\mathrm{H} 6 \mathrm{~A}-\mathrm{C} 6-\mathrm{H} 6 \mathrm{~B}$ & 108.2 & $\mathrm{C} 15-\mathrm{C} 16-\mathrm{H} 16 \mathrm{~A}$ & 119.7 \\
\hline $\mathrm{C} 1-\mathrm{C} 7-\mathrm{C} 10$ & $109.44(19)$ & $\mathrm{N} 1-\mathrm{C} 17-\mathrm{C} 16$ & $121.23(16)$ \\
\hline $\mathrm{C} 1-\mathrm{C} 7-\mathrm{H} 7 \mathrm{~A}$ & 109.8 & $\mathrm{~N} 1-\mathrm{C} 17-\mathrm{H} 17 \mathrm{~A}$ & 119.4 \\
\hline $\mathrm{C} 10-\mathrm{C} 7-\mathrm{H} 7 \mathrm{~A}$ & 109.8 & $\mathrm{C} 16-\mathrm{C} 17-\mathrm{H} 17 \mathrm{~A}$ & 119.4 \\
\hline $\mathrm{C} 11-\mathrm{C} 1-\mathrm{C} 2-\mathrm{C} 3$ & $177.81(19)$ & $\mathrm{C} 3-\mathrm{C} 8-\mathrm{C} 10-\mathrm{C} 7$ & $61.0(3)$ \\
\hline $\mathrm{C} 7-\mathrm{C} 1-\mathrm{C} 2-\mathrm{C} 3$ & $-59.4(2)$ & $\mathrm{C} 1-\mathrm{C} 7-\mathrm{C} 10-\mathrm{C} 9$ & $60.2(2)$ \\
\hline $\mathrm{C} 6-\mathrm{C} 1-\mathrm{C} 2-\mathrm{C} 3$ & $59.5(2)$ & $\mathrm{C} 1-\mathrm{C} 7-\mathrm{C} 10-\mathrm{C} 8$ & $-60.5(3)$ \\
\hline $\mathrm{C} 1-\mathrm{C} 2-\mathrm{C} 3-\mathrm{C} 4$ & $-60.9(3)$ & $\mathrm{C} 7-\mathrm{C} 1-\mathrm{C} 11-\mathrm{O} 1$ & $40.2(3)$ \\
\hline $\mathrm{C} 1-\mathrm{C} 2-\mathrm{C} 3-\mathrm{C} 8$ & $60.3(3)$ & $\mathrm{C} 2-\mathrm{C} 1-\mathrm{C} 11-\mathrm{O} 1$ & $162.52(19)$ \\
\hline $\mathrm{C} 8-\mathrm{C} 3-\mathrm{C} 4-\mathrm{C} 5$ & $-59.1(3)$ & $\mathrm{C} 6-\mathrm{C} 1-\mathrm{C} 11-\mathrm{O} 1$ & $-78.1(2)$ \\
\hline $\mathrm{C} 2-\mathrm{C} 3-\mathrm{C} 4-\mathrm{C} 5$ & $61.3(3)$ & $\mathrm{C} 7-\mathrm{C} 1-\mathrm{C} 11-\mathrm{C} 12$ & $-144.55(17)$ \\
\hline $\mathrm{C} 3-\mathrm{C} 4-\mathrm{C} 5-\mathrm{C} 9$ & $58.7(2)$ & $\mathrm{C} 2-\mathrm{C} 1-\mathrm{C} 11-\mathrm{C} 12$ & $-22.2(2)$ \\
\hline $\mathrm{C} 3-\mathrm{C} 4-\mathrm{C} 5-\mathrm{C} 6$ & $-61.2(3)$ & $\mathrm{C} 6-\mathrm{C} 1-\mathrm{C} 11-\mathrm{C} 12$ & $97.25(18)$ \\
\hline $\mathrm{C} 4-\mathrm{C} 5-\mathrm{C} 6-\mathrm{C} 1$ & $60.4(2)$ & $\mathrm{C} 17-\mathrm{N} 1-\mathrm{C} 12-\mathrm{C} 11$ & $-122.92(17)$ \\
\hline $\mathrm{C} 9-\mathrm{C} 5-\mathrm{C} 6-\mathrm{C} 1$ & $-59.9(2)$ & $\mathrm{C} 13-\mathrm{N} 1-\mathrm{C} 12-\mathrm{C} 11$ & $58.3(2)$ \\
\hline $\mathrm{C} 11-\mathrm{C} 1-\mathrm{C} 6-\mathrm{C} 5$ & $178.04(16)$ & $\mathrm{O} 1-\mathrm{C} 11-\mathrm{C} 12-\mathrm{N} 1$ & $0.6(3)$ \\
\hline $\mathrm{C} 7-\mathrm{C} 1-\mathrm{C} 6-\mathrm{C} 5$ & $59.4(2)$ & $\mathrm{C} 1-\mathrm{C} 11-\mathrm{C} 12-\mathrm{N} 1$ & $-174.80(15)$ \\
\hline $\mathrm{C} 2-\mathrm{C} 1-\mathrm{C} 6-\mathrm{C} 5$ & $-59.5(2)$ & $\mathrm{C} 17-\mathrm{N} 1-\mathrm{C} 13-\mathrm{C} 14$ & $0.7(3)$ \\
\hline $\mathrm{C} 11-\mathrm{C} 1-\mathrm{C} 7-\mathrm{C} 10$ & $-175.80(19)$ & $\mathrm{C} 12-\mathrm{N} 1-\mathrm{C} 13-\mathrm{C} 14$ & $179.53(17)$ \\
\hline $\mathrm{C} 2-\mathrm{C} 1-\mathrm{C} 7-\mathrm{C} 10$ & $59.3(2)$ & $\mathrm{N} 1-\mathrm{C} 13-\mathrm{C} 14-\mathrm{C} 15$ & $-0.6(3)$ \\
\hline $\mathrm{C} 6-\mathrm{C} 1-\mathrm{C} 7-\mathrm{C} 10$ & $-59.2(2)$ & $\mathrm{C} 13-\mathrm{C} 14-\mathrm{C} 15-\mathrm{N} 2$ & $179.56(19)$ \\
\hline $\mathrm{C} 4-\mathrm{C} 3-\mathrm{C} 8-\mathrm{C} 10$ & $59.7(3)$ & $\mathrm{C} 13-\mathrm{C} 14-\mathrm{C} 15-\mathrm{C} 16$ & $0.1(3)$ \\
\hline $\mathrm{C} 2-\mathrm{C} 3-\mathrm{C} 8-\mathrm{C} 10$ & $-60.7(3)$ & $\mathrm{N} 2-\mathrm{C} 15-\mathrm{C} 16-\mathrm{C} 17$ & $-179.07(18)$ \\
\hline $\mathrm{C} 4-\mathrm{C} 5-\mathrm{C} 9-\mathrm{C} 10$ & $-59.4(3)$ & $\mathrm{C} 14-\mathrm{C} 15-\mathrm{C} 16-\mathrm{C} 17$ & $0.4(3)$ \\
\hline $\mathrm{C} 6-\mathrm{C} 5-\mathrm{C} 9-\mathrm{C} 10$ & $60.4(3)$ & $\mathrm{C} 13-\mathrm{N} 1-\mathrm{C} 17-\mathrm{C} 16$ & $-0.2(3)$ \\
\hline $\mathrm{C} 5-\mathrm{C} 9-\mathrm{C} 10-\mathrm{C} 8$ & $60.2(3)$ & $\mathrm{C} 12-\mathrm{N} 1-\mathrm{C} 17-\mathrm{C} 16$ & $-179.00(16)$ \\
\hline $\mathrm{C} 5-\mathrm{C} 9-\mathrm{C} 10-\mathrm{C} 7$ & $-60.7(3)$ & $\mathrm{C} 15-\mathrm{C} 16-\mathrm{C} 17-\mathrm{N} 1$ & $-0.4(3)$ \\
\hline $\mathrm{C} 3-\mathrm{C} 8-\mathrm{C} 10-\mathrm{C} 9$ & $-59.9(3)$ & & \\
\hline
\end{tabular}

Hydrogen-bond geometry $\left(\AA,{ }^{\circ}\right)$

$\mathrm{Cg} 1$ is the centroid of the $\mathrm{N} 1 / \mathrm{C} 13-\mathrm{C} 17$ ring.

\begin{tabular}{lllll}
\hline$D-\mathrm{H} \cdots A$ & $D-\mathrm{H}$ & $\mathrm{H} \cdots A$ & $D \cdots A$ & $D-\mathrm{H} \cdots A$ \\
\hline $\mathrm{N} 2-\mathrm{H} 1 N 2 \cdots \mathrm{Br} 1^{\mathrm{i}}$ & $0.84(2)$ & $2.73(2)$ & $3.499(2)$ & $153(2)$ \\
$\mathrm{N} 2-\mathrm{H} 2 N 2 \cdots \mathrm{Br} 1^{\mathrm{ii}}$ & $0.85(2)$ & $2.56(2)$ & $3.393(2)$ & $169(2)$ \\
$\mathrm{C} 12-\mathrm{H} 12 A \cdots \mathrm{Br} 1^{\mathrm{ii}}$ & 0.97 & 2.72 & $3.664(2)$ & 166 \\
$\mathrm{C} 17-\mathrm{H} 17 A \cdots \mathrm{O} 1^{\mathrm{iv}}$ & 0.93 & 2.59 & $3.434(2)$ & 150 \\
$\mathrm{C} 14-\mathrm{H} 14 A \cdots C g 1^{\mathrm{i}}$ & 0.93 & 2.94 & $3.608(2)$ & 130
\end{tabular}

Symmetry codes: (i) $-x, y-1 / 2,-z+1 / 2$; (ii) $-x,-y+1,-z+1$; (iii) $x, y-1, z$; (iv) $x,-y+1 / 2, z+1 / 2$. 